\title{
Site investigation using EKS groundwater flow pathway images
}

\author{
R H Clarke and J W A Millar
}

GroundFlow Ltd, 29 High St. Marlborough Wiltshire SN8 1LW UK

\section{INTRODUCTION}

We report the development and use of equipment which images groundwater distribution and the water flow pathways in aquifers. Aquifer permeability profiles and maps are obtained from electrokinetic signals using algorithms based upon theoretical and laboratory studies (Chandler, 1971, Nourbehecht 1963).

Electrokinetic images reveal the characteristics of aquifers at a scale intermediate between the details obtainable from boreholes and the coarser grid used in aquifer modelling procedures. Their resolution is determined by the properties of the acoustic pulse used for making soundings. The electrical signals are produced at rock interfaces, which often allows both the depth and thickness of aquifers to be mapped. The equipment has been used successfully in a variety of environments including the English Chalk, unconsolidated and consolidated sand aquifers, tropical weathered basement aquifers, and a variety of desert aquifer types.

\section{METHOD}

We acquire electrokinetic signals (EKS signals) with a short, balanced dipole pair, using a patented arrangement to reference the signals (Figure 1). This arrangement produces a $70 \mathrm{~dB}$ improvement in signal to noise ratio and makes it practical for the first time to extract useful information from signals.

When an EKS observation is made the ground is struck at the shot point with a weight. A pressure wave travels into the ground from the point of impact and generates electrokinetic signals at each interface separating saturated porous materials of differing electrokinetic coefficient. A head wave also travels outwards from the shot point at the surface and from beneath the shot point along interfaces, like the ripples around a stone thrown into water. The subsurface head waves generate EKS signals which can be observed with an antenna lying directly overhead. Head wave signals show moveout. That is, their time of arrival varies with the distance from the antenna to the shot point; the "offset", according to the speed of travel of the head wave. Where the aquifer conditions are poor, as in the example, the head wave signals are weak and die out rapidly. This allows the signals formed by the pressure wave intersecting aquifer interfaces in the fresnel zone to be seen clearly, though weakly, at wide offsets. Such signals show no moveout, reaching all antennas simultaneously because the electrical signal returns to the surface from beneath the shot point at the speed of light. The EKS signals are, however, very weak except beneath the shot point.

Depth capability and resolution are determined by the nature of the seismic source and the characteristics of the weathering layer, as with seismic reflection surveys. EKS also shares with seismic a difficulty in imaging high energy or chaotic rock units, including steeply dipping bedded rocks, unweathered fractured basement, karst weathered carbonates, and alluvial fan conglomerates. 


\section{RESULTS}

Figure 2 shows unfiltered, unstacked, true-amplitude EKS and figure 3, simultaneous verticalmotion geophone signals. The EKS and geophone traces bear only a superficial resemblance to each other. The EKS data has a broader bandwidth and shows several flat (zero moveout) events, at 5 to $10 \mathrm{~ms}, 27$ to $30 \mathrm{~ms}$, and around $40 \mathrm{~ms}$, for example. These signals travel to the antenna from interfaces intersected by the downgoing p-wave at the speed of light and are visible because of the rapid attenuation of possible head wave signals which appear on the inner three traces (on the left). The signals were detected at 1 to $9 \mathrm{~m}$ offsets simultaneously on both sides of the shot point, that is, at up to $18 \mathrm{~m}$ apart. The plots are of the average signal from the two antennas. The data in figures 2 and 3 are from an outcrop of the Oxford Clay and Kellaways Beds Formations of the English Jurassic. Similar trials on saturated aquifers show much stronger EKS signals but the stronger moveout events associated with the aquifer interfaces obscure the weak zero-moveout events.

Head wave signals can obscure EKS signals but are not present in data recorded with GroundFlow's system which has a very small offset and records only the response to p-waves. This feature, together with the power line noise rejection capability, give GroundFlow's equipment its ability to image aquifer properties. Our results confirm part of a recently published model but suggest that, contrary to their expectations, shear wave conversion is not observed (Mikhailov, et al., 1997, figs. 2, 11).

Our data also show that, in certain conditions, the electrokinetic signal from successive interfaces can be used to image the dispersion and attenuation of the outgoing p-wave. Furthermore the EKS moveout events on multiple offset tests give the seismic velocity of the faster layer. Such information is likely to prove useful in seismic reflection surveying and we expect EKS signal recording to become a routine part of such work for the additional information it conveys.

GroundFlow and its clients have conducted EKS soundings at several thousand locations in Europe, Africa, the Middle East and Asia. Good results have been obtained in bedded sandstone and carbonate aquifers, in superficial deposits and in weathered basement (regolith) aquifers. Figure 4 shows a short profile across a sand-filled channel in Quaternary deposits. Such profiles reveal not only where boreholes might be drilled but how deep they should be and the extent of the aquifer which is likely to be accessible.

A simple algorithm provided with the EKS equipment allows information from soundings to be converted into borehole flow predictions. Such predictions have been used in Africa to manage groundwater supply drilling programmes. Figure 5 shows the comparison obtained between a number of EKS predictions and the actual flows from the corresponding boreholes.

\section{REFERENCES}

Chandler, R. N., 1981, Flow permeability from transient streaming potential measurements: SPE of AIME unsolicited conference paper SPE-9824.

Mikhailov, O.V., M.W. Haartsen, \& M.N. Toksoz, 1997, Electroseismic investigation of the shallow subsurface: field measurements and numerical modelling. Geophysics 62/1, 97-105.

Nourbehecht, B., 1963, Irreversible thermodynamic effects in inhomogeneous media and their applications in certain geoelectric problems: $\mathrm{PhD}$ thesis, Mass. Inst. of Technology. 
FIGURE 1 EKS survey equipment

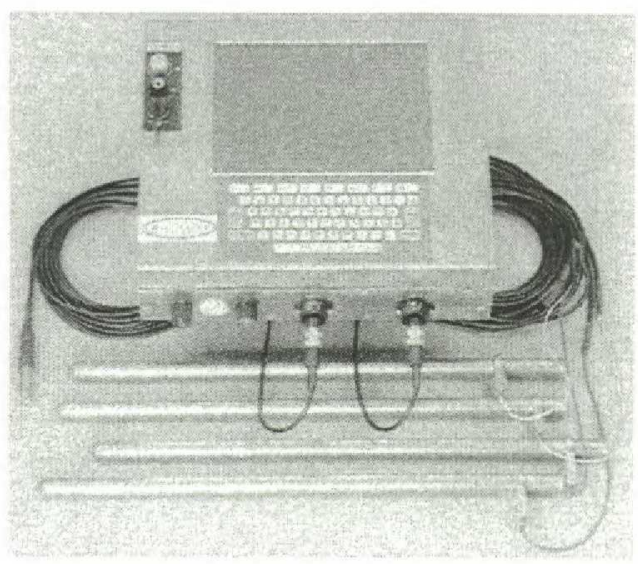

FIGURE 2 Offset Test at Foxham

EKS signal from $1 \mathrm{~m}$ antenna, offset 1 to $9 \mathrm{~m}$, shot point on left

Electrokinetic Signal, one division $=100$ microvolts

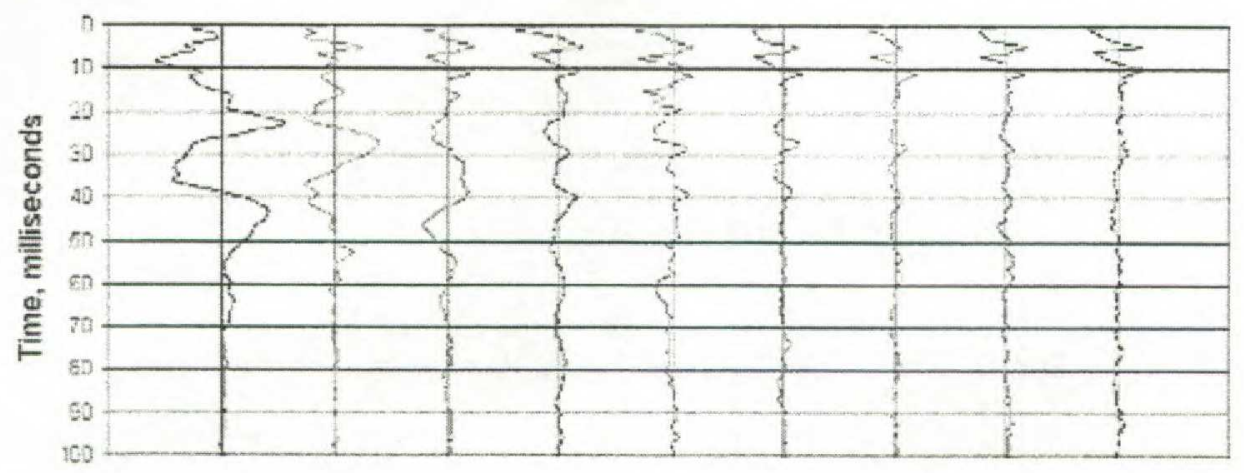

FIGURE 3 Offset test at Foxham

Simultaneous vertical motion geophone signal, offset 1 to $9 \mathrm{~m}$

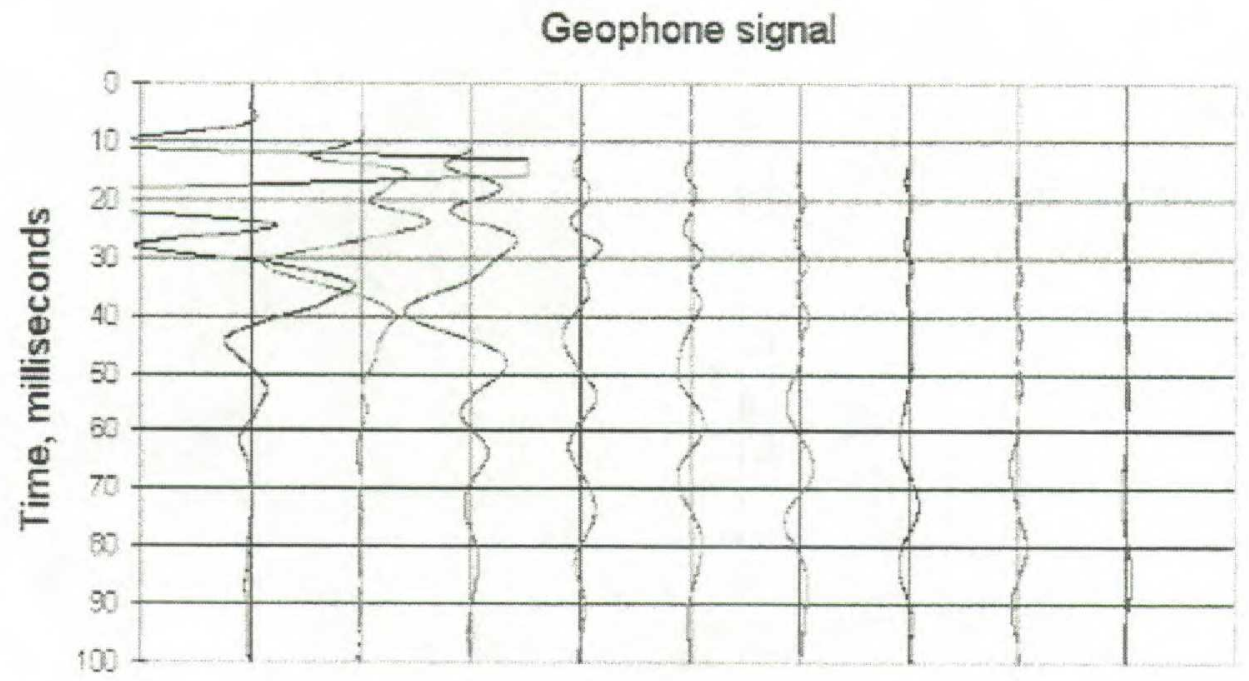


FIGURE 4 EKS Profile Across Quaternary Sand-filled Channels Vertical Scale 0 to $0.1 \mathrm{~s}$, one-way time, warm colours indicate higher permeability, vertical lines indicate shotpoints

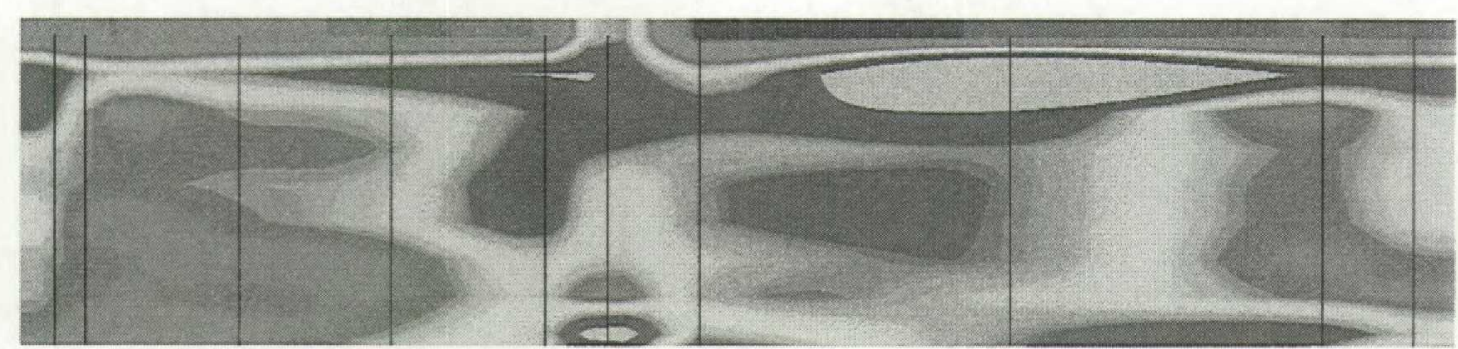

$100 \mathrm{~m}$

FIGURE 5 Comparison of Predicted and Actual Borehole Flow Rates

African Data

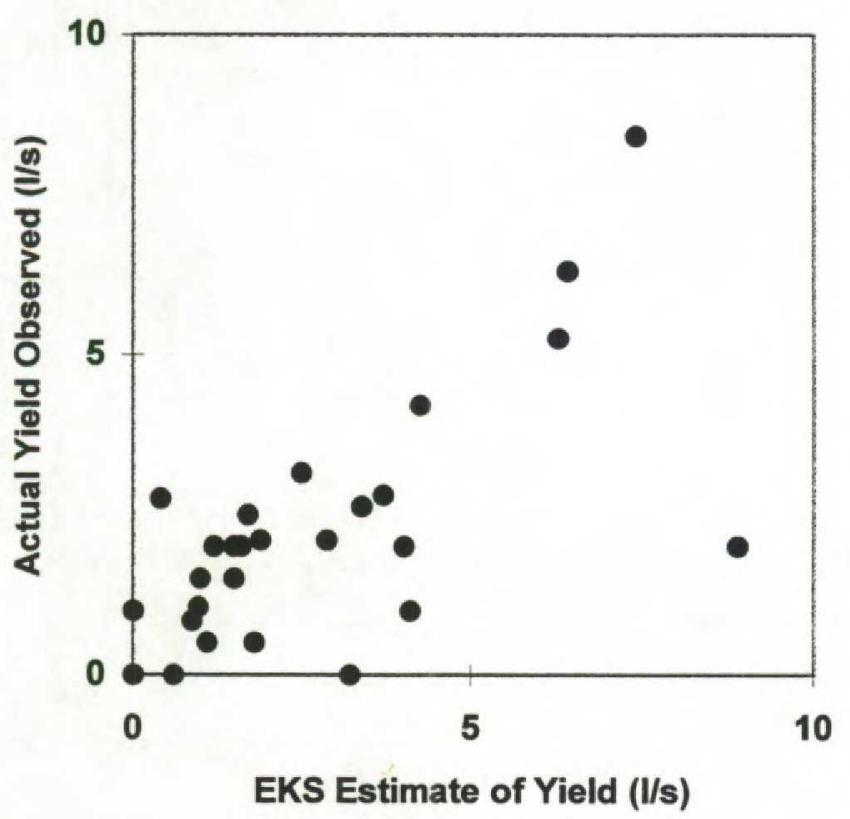

